\title{
Spontaneous Assembly of a Monolayer of Charged Gold Nanocrystals at the Water/Oil Interface
}

\author{
Francois Reincke, Stephen G. Hickey, Willem K. Kegel, \\ and Daniel Vanmaekelbergh*
}

Novel materials based on 2D and 3D arrays of nanocrystalline building blocks can be prepared from colloidal suspensions by controlled processing. There is a large effort in this field due to the exciting electrical, ${ }^{[1-3]}$ magnetic ${ }^{[4,5]}$ and optical ${ }^{[6,7]}$ properties of such systems, which can be tuned by the size of the building blocks and the interactions between them. Ordered arrays of uncharged, sterically stabilized nanocrystals have been successfully obtained by a subtle destabilization of the suspension ${ }^{[8]}$ or by assembly at a liquid/air ${ }^{[9-11]}$ or liquid/liquid interface. ${ }^{[12]}$ There is, however, an important class of suspensions that are stable owing to the nanocrystal surface charge. Assembly of uncapped, charged nanocrystals into arrays is considerably more difficult as destabilization of a suspension usually leads to uncontrolled coagulation. ${ }^{[13]}$ Herein, we report surprising results that may lead to novel routes for the controlled fabrication of materials from chargestabilized nanocrystal colloids. We have observed that gold nanocrystals spontaneously form a monolayer at the water/oil interface if the surface charge of the nanocrystals is gradually reduced. The separation between the nanocrystals in the layer is smaller than the width of the diffuse electrical double layer. Nevertheless, coagulation of the particles into clumps does not occur. The monolayers are remarkably robust and can be easily transferred to substrates, opening the way to technological applications. The spontaneous 2D assembly of charged nanocrystals is qualitatively described in terms of a reduction of the water/oil interfacial energy upon particle adsorption counteracted by electrostatic repulsion in the film.

Suspensions of sterically stabilized and supposedly uncharged gold nanocrystals have been used as model systems for the study of self-assembly. A wealth of gold nanocrystal structures have been reported $\mathrm{d}^{[9,11,14]}$ and their optoelectrical properties are still under investigation. The synthesis of charge-stabilized gold sols is well established. The processing of such sols has been focused on the capping of charged nanocrystals with organic molecules to allow transfer to a

[*] F. Reincke, Dr. S. G. Hickey, ${ }^{+}$Prof. Dr. D. Vanmaekelbergh Physics and Chemistry of Condensed Matter

Debye Institute, Utrecht University, 3508 TA Utrecht

(The Netherlands)

Fax: $(+31)$ 30-253-2403

E-mail:daniel@phys.uu.nl

Dr. W. K. Kegel

van't Hoff Laboratory for Physical and Colloid Chemistry

Debye Institute, Utrecht University, 3508 TA Utrecht The Netherlands)

$\left.{ }^{+}\right]$Present address:

Institut für Physikalische Chemie, Universität Hamburg

Bundesstrasse 45, 20146 Hamburg (Germany) 
nonaqueous phase. In addition, the assembly of these nanocrystals into molecular-like structures, covalently linked by organic bridges ${ }^{[15]}$ or DNA strands ${ }^{[16]}$ have been reported. Herein, we report the formation of an interfacial monolayer of charged gold nanocrystals from an aqueous gold sol upon gradual reduction of the surface charge.

Aqueous suspensions of charge-stabilized gold nanocrystals with diameters between 8 and $40 \mathrm{~nm}$ were prepared according to the method of Frens. ${ }^{[17]}$ The gold nanocrystals in the suspension have a negative surface charge due to the citrate or gold-chloride anions from the gold surface. About half the original charge density is preserved upon further addition of ethanol, and the gold sol remains stable. However, when a layer of heptane is introduced above this solution, gold nanocrystals quickly adsorb at the water/heptane interface. A vial containing the alcohol-free aqueous gold sol (pink) covered with an immiscible layer of heptane (colorless) is shown in Figure $1 \mathrm{~b}$ (left). Upon addition of ethanol to the gold sol, a blue layer with a metallic aspect is immediately formed at the water/oil interface (Figure 1b, centre and
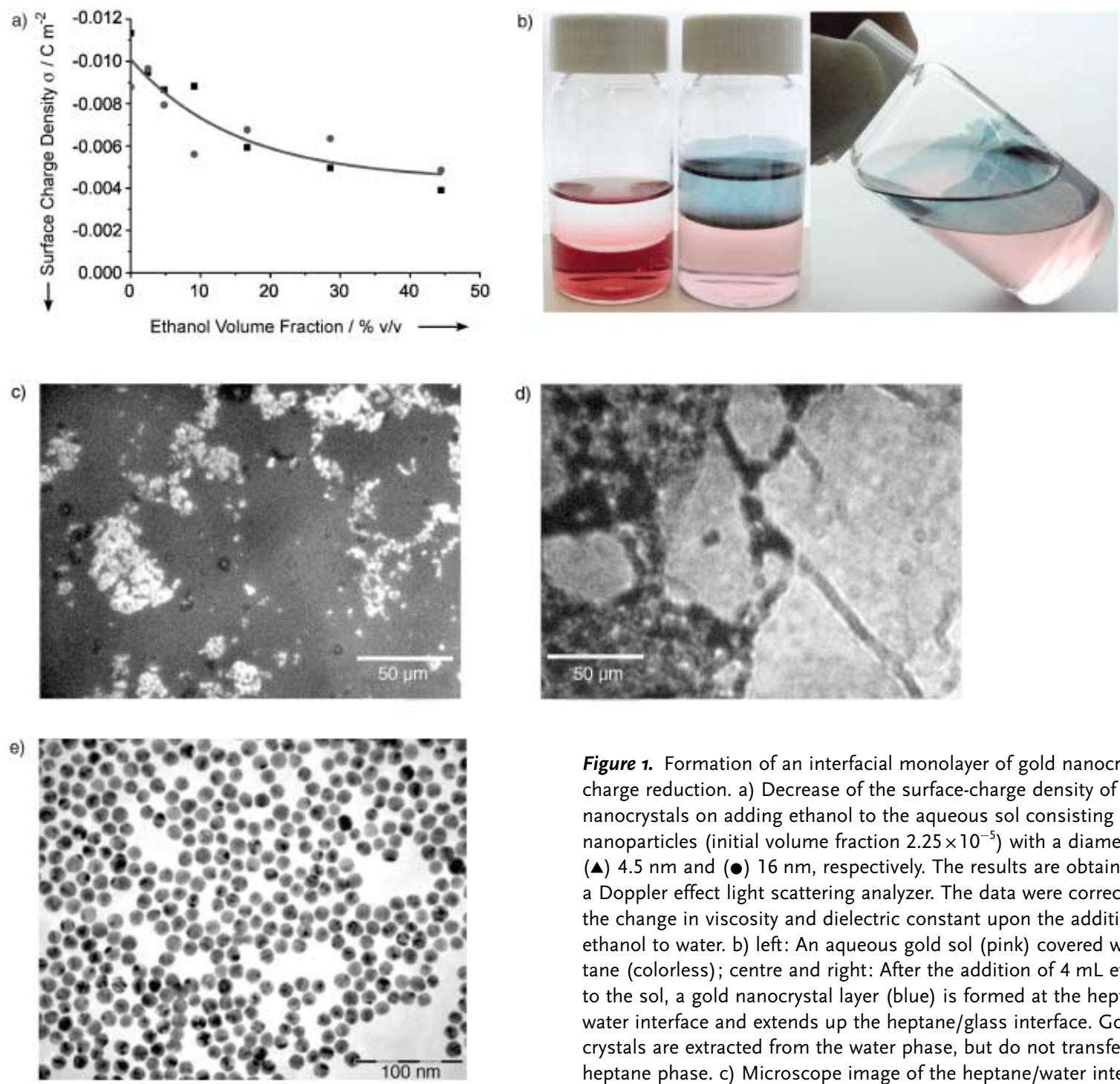

adsorption of citrate and gold-chloride anions. ${ }^{[18]}$ We determined the surface-charge density by measuring of the nanocrystal diffusion coefficient with an ac-electrophoresis method (Figure 1a). The as-prepared gold nanocrystals have a surface-charge density of about $-0.010 \mathrm{Cm}^{-2}$. It can be seen in Figure $1 \mathrm{a}$ that the addition of ethanol to the sol gradually decreases the surface charge, very likely because of competitive adsorption of ethanol molecules ${ }^{[19]}$ which displace the

Figure 1. Formation of an interfacial monolayer of gold nanocrystals by charge reduction. a) Decrease of the surface-charge density of the gold nanocrystals on adding ethanol to the aqueous sol consisting of $\mathrm{Au}$ nanoparticles (initial volume fraction $2.25 \times 10^{-5}$ ) with a diameter of (A) $4.5 \mathrm{~nm}$ and $(\bullet) 16 \mathrm{~nm}$, respectively. The results are obtained with a Doppler effect light scattering analyzer. The data were corrected for the change in viscosity and dielectric constant upon the addition of ethanol to water. b) left: An aqueous gold sol (pink) covered with heptane (colorless); centre and right: After the addition of $4 \mathrm{~mL}$ ethanol to the sol, a gold nanocrystal layer (blue) is formed at the heptane/ water interface and extends up the heptane/glass interface. Gold nanocrystals are extracted from the water phase, but do not transfer to the heptane phase. c) Microscope image of the heptane/water interface after addition of $1.7 \mathrm{~mL}$ ethanol to $10 \mathrm{~mL}$ of gold sol covered with $5 \mathrm{~mL}$ of heptane: the coverage is low and islands of gold nanocrystals are visible. d) Microscope image of the heptane/water interface after addition of $3.8 \mathrm{~mL}$ of ethanol to $10 \mathrm{~mL}$ of gold sol: the gold nanocrystals form large domains $(>50 \mu \mathrm{m})$ that consist of a dense layer of nanocrystals. e) TEM image of a layer with maximum coverage (65\%) collected from the heptane/water interface by a Langmuir-Blodgett technique showing that the layer does not contain $3 \mathrm{D}$ aggregates, the voids have a typical size equivalent to $1-10$ nanocrystals; the space between particles is $1-4 \mathrm{~nm}$. 
right). The nanocrystal coverage of the water/oil interface increases gradually with the decrease of the surface charge density on the gold nanocrystals. Figure $1 \mathrm{c}$ and $1 \mathrm{~d}$ shows in situ optical microscope pictures of the nanocrystal layer after addition of 17 and 38 volume $\%$ of ethanol, respectively. Upon the addition of $17 \%$ of ethanol, islands of gold nanocrystals form (Figure 1c); they extend and coalesce to a coherent monolayer as the ethanol content increases (Figure 1d). The monolayers do not collapse into multilayers nor are the gold nanocrystals transferred to the heptane phase. Instead, the layer creeps up the glass wall of the vial (see Figure $1 \mathrm{~b}$, centre and right). This indicates that there is a strong driving force for the formation of an interfacial monolayer. The films are remarkably robust if one considers that the monolayer reaches up the vertical wall of the vial to a height of a few centimeters without breaking into pieces (see Figure $1 \mathrm{~b}$, centre and right). The interfacial nanocrystal layers could be transferred to a glass or mica substrate as a Langmuir-Blodgett film, and studied by atomic force microscopy (AFM) and transmission electron microscopy (TEM); a typical TEM image is presented in Figure $1 \mathrm{e}$. In all cases we found monolayers of gold nanocrystals; coagulation of the gold nanocrystals into 3D aggregates and the spontaneous collapse of the film into multilayers was not observed. We checked that the structure of the interfacial gold nanocrystal layer is not disturbed by the transfer procedure, which is commonly the case for Langmuir-Blodgett transfer. ${ }^{[9]}$ The nanocrystal coverage of the water/oil interface saturates at around $65 \%$ for $16 \mathrm{~nm}$ particles. Similar results were obtained for gold crystals with a diameter in the range 8 to $40 \mathrm{~nm}$. The typical distance between the nanocrystal surfaces was between 1 and $4 \mathrm{~nm}$ (Figure 1e). This is smaller than twice the width of the electrical double layer around the gold nanocrystals in the sol $(2 \times 5 \mathrm{~nm})$. The layers are not ordered, which may arise from the size dispersion of the nanocrystals being about $10 \%$. The layers have voids equivalent to, typically, between one and ten nanocrystals. The metallic sheen of the monolayer is due to electronic coupling between the uncapped nanocrystals. ${ }^{[20,21]}$

Adsorption of a charged gold nanocrystal at the water/oil interface must be driven by a reduction of the interfacial energy; the repulsion between the charged nanocrystals in the monolayer forms a counteracting force. We conjecture that the reduction of the charge density on the gold nanocrystals is a prerequisite for the formation of the nanocrystal monolayers at the water/oil interface. This is supported by experiments in which hydrochloric or sulfuric acid was added to the sol to lower the $\mathrm{pH}$ from about 5.8 to 2.0. As a result, the charge on the gold surface decreases by protonation of the adsorbed citrate ions. As for ethanol addition, monolayers of gold nanocrystals were formed at the water/oil interface. Again, coagulation does not occur. In contrast, a decrease in the thickness of the diffuse electrical double layer by adding salt to the gold sol leads to coagulation in the bulk solution. Thus, a decrease in the charge density of the nanocrystals leads to the controlled formation of a monolayer of gold nanocrystals at the water/oil interface, while reduction of the width of the diffuse double layer leads to conventional destabilization $^{[14]}$ of the suspension.
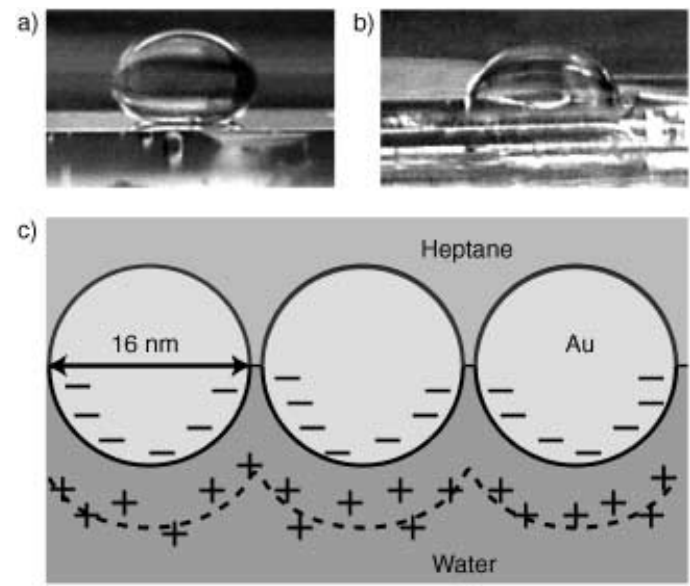

Figure 2. Wetting of an uncharged and charged gold surface with a drop of a mixture of water and ethanol covered with a heptane phase. a) A water $+37 \%$ ethanol droplet immersed in heptane shows a negative contact angle with flame-annealed gold, which shows that heptane has a stronger affinity for uncharged gold than the water droplet. b) As in (a) but now with a gold surface on which citrate has been adsorbed (the gold substrate has been immersed in an aqueous solution of $1 \% \mathrm{v} / \mathrm{v}$ trisodium citrate for 1 hour): the water + ethanol droplet shows a contact angle of $90^{\circ}$, which indicates a similar affinity of the water mixture and heptane phase for charged, citrate-covered gold c) Schematic representation of the nanocrystals at the heptane/water interface, derived from the experiment described by (b), and TEM and AFM characterization. The charged gold nanocrystals are half in the water and half in the heptane phase. Most charge is located at the water side, but charge remaining at the gold/oil interface determines the degree of Coulomb repulsion, see text.

The energy of the layer of charged gold nanocrystals adsorbed at the water/heptane interface is decided by three quantities; a) the interfacial energy due to surface tension, b) the electrostatic energy and c) van der Waals attraction between the nanocrystals. These contributions are discussed below.

a) The first contribution is related to the surface tension of the water/oil $\left(\chi_{\mathrm{w} / \mathrm{o}}\right)$, nanocrystal/water $\left(\chi_{\mathrm{c} / \mathrm{w}}\right)$ and nanocrystal/oil $\left(\chi_{c / o}\right)$ interface. The microscopic structure of the interfacial nanocrystal layer is of key importance for a valuable estimation of this contribution. We, therefore, studied the wetting of the (charged gold/water and ethanol/heptane) three-phase system, see Figure 2. With flame-annealed gold the contact angle is negative (Figure $2 \mathrm{a}$ ), thus showing that heptane has a stronger affinity for hydrophobic gold than the drop of water and ethanol. This changed significantly when citrate was adsorbed on the gold. We found that the contact angle $\theta$ between heptane at a charged gold substrate and a drop of a mixture of water and ethanol is close to 90 degrees (Figure 2b). According to the Young-Dupré equation $\cos \theta=\left(\chi_{\mathrm{c} / \mathrm{o}}-\chi_{\mathrm{c} / \mathrm{w}}\right) / \chi_{\mathrm{w} / \mathrm{o}}$ thus $\chi_{\mathrm{c} / \mathrm{o}} \cong \chi_{\mathrm{c} / \mathrm{w}}$, in which c represents the charged gold, w represents the aqueous phase, o represents the oil phase, and these / represents the interface. This means that the gold particles are half in the oil and half in the water phase (Figure 2c). Adsorption of a gold particle at the water/oil interface leads to a reduction in the interfacial energy related to the surface 
tension of roughly $-\pi R^{2} \chi_{\mathrm{w} / \mathrm{o}}, R$ is the radius of the gold nanocrystal. For example, with gold nanocrystals with a diameter of $16 \mathrm{~nm}$ and $\chi_{\mathrm{w} / \mathrm{o}}$ being $5 \mathrm{mN} \mathrm{m}^{-1}$, this amounts to $-240 \mathrm{kT}$ per adsorbed particle. This contribution is independent of the nanocrystal interfacial coverage.

b) The main force counteracting layer formation is electrostatic repulsion between the negatively charged nanocrystals in the layer. This depends strongly on the distribution of the charge over the surface of the gold nanocrystal, which is half in the water and half in the oil phase. Very probably, there is a considerable redistribution of the remaining surface charge when the particle is adsorbed at the interface. In addition, the diffuse ion cloud at the water side could become considerably deformed. Residual charges at the oil side of the water/ oil interface lead to unscreened Coulombic repulsion between the adsorbed particles. ${ }^{[22]}$

c) The third contribution to the total energy is due to van der Waals interactions between the gold nanocrystals. This contribution becomes more important with increasing nanocrystal coverage, and thus counteracts the effect of Coulombic repulsion to a certain extent. We remark that van der Waals interactions are not the main driving force for layer formation. If van der Waals interactions were predominant, 3D coagulation would prevail.

A quantitative model for adsorption of charged nanocrystals at the water/oil interface will be presented in detail elsewhere. Here we show the nanocrystal coverage of the water/oil interface as a function of the decreasing effective charge density on the nanocrystals calculated on the basis of the contributions discussed above (Figure 3). The interfacial coverage is small at the maximum charge density (no ethanol added) and increases gradually with a reduction of the charge density. Thus, the crude model is in agreement with our observation that, although layer formation is not observed with the as-prepared gold sol, a decrease of the charge density on the gold nanocrystals by a factor of two leads to the formation of an interfacial layer with a significant nanocrystal coverage (about 65\%). The model also explains layer formation in a qualitatively similar way for a wide range of particle sizes as observed experimentally. The inhomogeneous microscopic structure of the nanocrystal monolayer is more difficult to explain: it is not clear yet why dense 2D domains coexist with relatively large voids. Possibly, the van der Waals interactions which favor dense layers compete, on a scale of 100 nanometer, with Coulombic repulsion; this may lead to 2D aggregates of a limited lateral size coexisting with voids, in a similar way as has been shown in the $3 \mathrm{D}$ case. $^{[23]}$

Charged colloidal nanocrystals have considerable technological relevance. Many preparation methods lead to chargestabilized sols. Furthermore, it has become clear that even sterically stabilized colloids in nonaqueous solvents can carry residual charge. ${ }^{[2]}$ The preparation of monolayers of such charged colloids would form an important step forwards in the route to the fabrication of $2 \mathrm{D}$ and $3 \mathrm{D}$ materials for optoelectrical or magnetic applications. We have shown that charged gold nanocrystals self-assemble at the water/oil

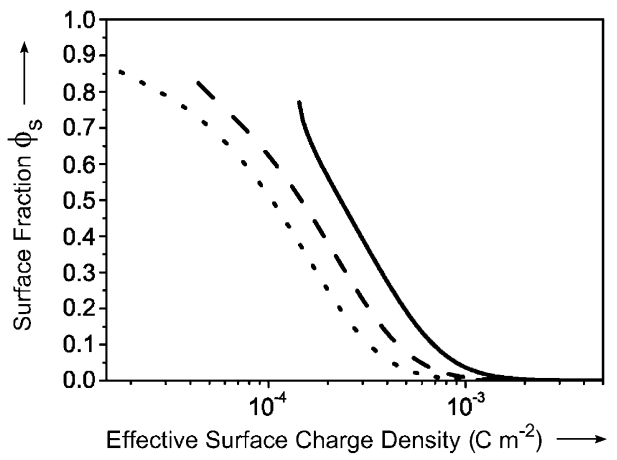

Figure 3. The gold nanocrystal coverage of the water/oil interface as a function of the effective charge density on the nanocrystals. The results are obtained from a model that accounts for the reduction of the water/oil surface tension, and the repulsion and van der Waals attraction between the charged nanocrystals (see text). The equilibrium coverage increases with decreasing surface-charge density for all sizes of nanocrystals (full line: $16 \mathrm{~nm}$ diameter; dashed line: $40 \mathrm{~nm}$ diameter, dotted line: $80 \mathrm{~nm}$ diameter). Details of the model will be presented elsewhere.

interface upon gradual reduction of the surface charge density. As charge reduction can be accomplished in various ways, this method of assembly does not depend on the details of the surface chemistry and should thus be widely applicable. Our discovery of spontaneous monolayer formation of charged gold nanocrystals at the water/oil interface forms a new impetus to research devoted to the controlled 2D assembly of charged nanocrystals.

Received: July 9, 2003

Revised: October 22, 2003 [Z52339]

Keywords: colloids - electrostatic interactions - interfaces . nanostructures · self-assembly

[1] M. Brust, D. Bethell, D. J. Schiffrin, C. J. Kiely, Adv. Mater. 1995, 7, 795 .

[2] R. Parthasarathy, X. M. Lin, H. M. Jaeger, Phys. Rev. Lett. 2001, $87,186807$.

[3] A. L. Roest, J. J. Kelly, D. Vanmaekelbergh, E. A. Meulenkamp, Phys. Rev. Lett. 2002, 89, 36801.

[4] H. Zeng, J. Li, J. P. Liu, Z. L. Wang, S. H. Sun, Nature 2002, 420, 395.

[5] C. B. Murray, S. H. Sun, W. Gaschler, H. Doyle, T. A. Betley, C. R. Kagan, IBM J. Res. Dev. 2001, $45,47$.

[6] C. R. Kagan, C. B. Murray, M. Nirmal, M. G. Bawendi, Phys. Rev. Lett. 1996, 76, 1517.

[7] S. A. Crooker, J. A. Hollingsworth, S. Tretiak, V. I. Klimov, Phys. Rev. Lett. 2002, 89, 186802.

[8] C. B. Murray, C. R. Kagan, M. G. Bawendi, Science 1995, 270, 1335.

[9] J. R. Heath, C. M. Knobler, D. V. Leff, J. Phys. Chem. B 1997, $101,189$.

[10] R. P. Andres, J. D. Bielefeld, J. I. Henderson, D. B. Janes, V. R. Kolagunta, C. P. Kubiak, W. J. Mahoney, R. G. Osifchin, Science 1996, 273, 1690.

[11] C. J. Kiely, J. Fink, M. Brust, D. Bethell, D. J. Schiffrin, Nature 1998, 396, 444. 


\section{Communications}

[12] Y. Lin, H. Skaff, T. Emrick, A. D. Dinsmore, T. P. Russell, Science 2003, 299, 226.

[13] E. J. W. Verwey, J. T. G. Overbeek, Theory of the Stabiliy of Lyophobic Colloids, Dover, New York, 1999.

[14] J. Fink, C. J. Kiely, D. Bethell, D. J. Schiffrin, Chem. Mater. 1998 $10,922$.

[15] J. P. Novak, D. L. Feldheim, J. Am. Chem. Soc. 2000, 122, 3979.

[16] J. J. Storhoff, C. A. Mirkin, Chem. Rev. 1999, 99, 1849.

[17] G. Frens, Nature 1973, 241, 20.

[18] M. K. Chow, C. F. Zukoski, J. Colloid Interface Sci. 1994, 165, 97.

[19] F. Reincke, S. G. Hickey, J. J. Kelly, T. W. Braam, L. W. Jenneskens, D. Vanmaekelbergh, J. Electroanal. Chem. 2002, $522,2$.

[20] C. P. Collier, R. J. Saykally, J. J. Shiang, S. E. Henrichs, J. R. Heath, Science 1997, 277, 1978.

[21] G. Markovich, C. P. Collier, J. R. Heath, Phys. Rev. Lett. 1998, 80 , 3807.

[22] R. Aveyard, B. P. Binks, J. H. Clint, P. D. I. Fletcher, T. S Horozov, B. Neumann, V. N. Paunov, J. Annesley, S. W. Botchway, D. Nees, A. W. Parker, A. D. Ward, A. N. Burgess, Phys. Rev. Lett. 2002, 88, 246102.

[23] J. Groenewold, W. K. Kegel, J. Phys. Chem. B 2001, 105, 11702.

[24] L. N. Donselaar, A. P. Philipse, J. Suurmond, Langmuir 1997, 13, 6018 